\title{
When Sound Stops: Offset Responses in the Auditory System
}

Conny Kopp-Scheinpflug ${ }^{1}$, James L. Sinclair ${ }^{2}$, and Jennifer F. Linden ${ }^{2,3, *}$

${ }^{1}$ Division of Neurobiology, Department Biology II, Ludwig-Maximilians-University, Munich, Germany

${ }^{2}$ Ear Institute, University College London, London, UK

${ }^{3}$ Department of Neuroscience, Physiology \& Pharmacology, University College London, London, UK

*Correspondence: j.linden@ucl.ac.uk (J.F. Linden).

\section{Keywords:}

hearing, sound, temporal processing, post-inhibitory rebound, ON/OFF asymmetries, gap detection

\begin{abstract}
The auditory modality is fundamentally a temporal sense, requiring analysis of changes in sound signals on timescales ranging from microseconds to minutes. To generate a faithful representation of changes in sound intensity and frequency over time, sound offsets (disappearances) as well as sound onsets (appearances) must be encoded by the auditory system. Here we review the computational significance, perceptual roles, anatomical locations, and cellular and network origins of sound-offset responses in the mammalian auditory brain. We show that sound-offset responses arise from mechanisms and pathways distinct from those producing sound-onset responses, and are likely to be essential for auditory processing of temporally discontinuous sounds such as speech.
\end{abstract}




\section{Sound-Offset Responses in the Auditory System}

Mechanoelectrical transduction mechanisms in the ear encode transient changes in sound pressure with sub-millisecond precision. The auditory nerve then provides to the brain a high-fidelity representation of sound frequency, intensity, and timing, usually including sharp increases in nerve activity following sound onsets and decreases following sound offsets [1]. Many neurons in the auditory brain also represent sound onsets and offsets in this way; however, others produce bursts of activity following sound offsets (e.g., [2]), or following both onsets and offsets (e.g., [3]).

Historically, most studies of the central auditory system have ignored sound-offset responses, or dismissed them on neurobiological, acoustical, or perceptual grounds. Neurobiologically, sound-onset responses are much more prevalent in the auditory system than sound-offset responses [4], especially in anaesthetized animals [5], in which the majority of in vivo studies have been performed. Acoustically, offsets of natural sounds tend to be less abrupt than onsets [6], and sound offsets are often obscured by reverberation, especially in enclosed environments. Lastly, perceptually, sound offsets are less salient than sound onsets $[4,7,8]$.

Nevertheless, neurons with sound-offset responses are found throughout the auditory system. These neurons appear to be especially concentrated in particular nuclei of the auditory brainstem [9] and specific subregions of the thalamus [10], suggesting the existence of a dedicated "offset pathway" in the auditory brain. Moreover, recent studies indicate that neural representation of sound transients, including offsets, may be key to perception of communication sounds such as speech $[1,11]$. It is time to re-assess the importance of sound-offset responses in the auditory system.

Here we define a sound offset as a rapid decline in sound intensity over no more than a few milliseconds, following a sustained sound of at least a few tens of milliseconds in duration (see Glossary). For the purposes of this Review, we also define a sound-offset response as an increase in neural activity that is tightly time-locked to a sound offset. We do not attempt to review the wider literature on perception and neural representation of amplitude modulation [1], or the ways in which interaction of 
excitation and inhibition over time can shape temporal filter characteristics of auditory neurons $[2,12,13]$. Nor do we comprehensively review reports of suppression of neural activity following sound offsets [14,15]. Although this post-stimulus suppression could be considered a form of sound-offset response, such suppression also commonly arises from more general adaptation mechanisms, including in the auditory nerve [16]. Our focus here is specifically on excitatory sound-offset responses, particularly those which might arise from dedicated cellular mechanisms or synaptic interactions in the central auditory pathway. 


\section{Computational Significance of Sound Offsets in Auditory Processing}

Segregated neural pathways for processing increments and decrements in stimulus intensity are common in sensory systems and a feature of many sensory computations ranging from motion detection in flies [17] to olfaction in nematodes [18]. The most well-known and intensively studied example is in the mammalian visual system, where ON and OFF pathways are established at the level of the retina, maintained in segregated channels through the lateral geniculate nucleus of the visual thalamus, and then integrated in a push-pull fashion in the visual cortex [19].

There are broad similarities between onset and offset responses in the mammalian auditory system and ON and OFF responses in the mammalian visual system, although the auditory system operates on a much faster timescale. Auditory brainstem nuclei, where segregated onset and offset responses first appear, are arguably analogous to neural circuits within the retina where the visual ON and OFF pathways form, in terms of polysynaptic distance from primary sensory receptors. Moreover, the vast majority of auditory cortical neurons with offset responses also exhibit onset responses [3], indicating that, as in the visual system, early segregation of onset and offset responses is followed by re-integration at or below the level of the cortex. However, onset and (especially) offset responses in the auditory system are strongly transient, and therefore are most analogous to the bursts of firing produced in visual ON and OFF pathways by appearances and disappearances of light, rather than the more sustained ON or OFF firing evoked by stable bright or dark patches. Thus, even more than visual ON and OFF responses, auditory onset and offset responses are neural representations of temporal change.

What are the computational advantages of splitting time-varying sensory input into two pathways representing opposite directions of change? The key advantage is likely to be metabolic efficiency: "saving spikes" for representation of intensity change $[19,20]$. Of course, a single-channel system (e.g., a differentiator) can represent change with transient positive and negative outputs for stimulus increments and decrements. However, spontaneous firing rates would need to be high to achieve equivalent dynamic range for representing increments and decrements of different 
magnitudes with increases or decreases in firing rate (Figure 1A). An ON-OFF system maximises information transfer while minimising spike rate, even in comparison with a dual-pathway ON-ON system [20].

Another computational advantage of segregated onset and offset pathways may be that biophysical implementation of behaviorally relevant nonlinear computations is simplified [20]. This principle is illustrated in the visual motion detection system of the fly, where sign-correct multiplication is achieved by splitting visual input into two excitatory ON and OFF pathways, and then combining successive signals within each pathway [17]. In the auditory system, combination of non-negative signals across onset and offset pathways is thought to contribute both to duration discrimination (Box 2) and gap detection [21] (see "Perceptual Roles of Sound Offsets").

Notably, most computational models of auditory cortical and thalamic responses cannot adequately account for the response properties of neurons with both onset and offset responses [7]. For example, spectrotemporal receptive field models cannot produce positive outputs for both onsets and offsets because the initial sound-filtering step is linear in the stimulus spectrogram. Related linear-nonlinear extensions, including those implementing adaptation or contrast gain control, are similarly limited if the output nonlinearity is constrained to be monotonic. Models incorporating non-monotonic output nonlinearities [22], input nonlinearities [23], or nonlinear excitatory-inhibitory networks [24] may be capable of capturing onset and offset responses with certain parameter settings, but these regimes have not been explored in previous studies. Successful modelling of offset as well as onset responses in auditory cortex or thalamus has primarily been achieved by explicitly modelling segregated onset- and offset-sensitive input channels [7,25,26]. 


\section{Perceptual Roles of Sound Offsets}

Sound offsets play important roles both in auditory scene analysis and in speech perception. Sound terminations act as temporal edges for perceptual grouping [27] and duration discrimination (see Box 2). Gaps in sound are also essential cues for consonant discrimination; for example, perceptual and neural discrimination of "da" and " $t a$ " is determined by whether the time between release of the stop consonant and onset of voicing is less or more than 40ms [28] (Figure 1B). Here, we consider the roles of sound offsets in termination detection and gap detection separately, to clarify key differences in the perceptual constraints.

The physics of sound production and reverberation makes detecting the termination of a sound more challenging than detecting its initiation. Many natural sounds are produced by impact-induced vibrations that decay, and therefore the intensity change of the sound source is more abrupt at onset than offset [6] (Figure 1C). Moreover, a sound wave arriving at the ear directly from a sound source will be superposed with sound waves arising from reverberation off walls and other objects in the environment. In a medium-sized room, sound reverberations arrive within a few milliseconds of sound onset, and therefore may obscure the offset of all but the very shortest sounds [29]. Thus, in the natural world, abrupt increases in sound intensity are more commonly experienced than abrupt decreases in sound intensity. In the visual system, onset/offset asymmetries in natural stimulus statistics have been linked to ON/OFF asymmetries in neural pathways and perception [30,31]. Similarly, evolutionary adaptation to the properties of natural sounds might explain why onset responses predominate in the auditory brain $[4,7,8]$. However, the existence of specialized mechanisms and pathways for generating offset responses (Figures 2 and 3 ) suggests that the auditory system evolved in part to meet the challenges of detecting sound termination in natural environments.

Sound offsets also serve as cues for gap detection. Thresholds for detection of gaps in otherwise continuous sounds are extremely short (2-3ms) in normal subjects, and therefore these within-channel gap-detection thresholds are often used as an objective measure of the limits of auditory temporal acuity [32]. When spectral 
disparity is introduced between the sounds leading and trailing the gap (between-channel gap detection), thresholds increase by an order of magnitude, to approximately 30ms [33]. Perceptually, between-channel gaps sound like interruptions between distinct events, while within-channel gaps sound more like "blips" in an ongoing noise, or momentary changes in sound texture. Both forms of gap detection are likely to be important for speech perception in noise: between-channel for discrimination of voice-onset time in consonants [33,34], and within-channel for glimpsing brief moments of clear speech signal otherwise masked by fluctuating background noise [35].

Higher thresholds for between-channel than within-channel gap detection suggest that the mechanisms of temporal correlation across sound frequencies differ from those of discontinuity detection. Studies in both humans and mice have already indicated that sound offsets play an important role in temporal correlation across sound frequencies [36-38]. Thus sound-offset responses seem likely to be crucial for between-channel gap detection. However, the contribution of sound-offset responses to within-channel gap detection remains unclear. In mice, optogenetic suppression of auditory cortical activity during the period immediately after a brief gap in noise (but not during the gap) disrupts gap detection, suggesting a primary role for neural activity during the post-gap (i.e., onset) period [39]. However, in an animal model of gap-detection deficits, auditory thalamic abnormalities in gap-in-noise sensitivity have been linked to specific deficits in offset responses, whereas onset responses appear normal [25] (Box 3). Moreover, in humans, cortical event-related potentials evoked by brief gaps in noise include a distinctive N1 component specifically related to the cessation of an ongoing sound [40]. Thus, the neural basis for gap detection remains in question. 


\section{Neural Mechanisms of Sound-Offset Responses}

Sound-offset responses have been observed throughout the auditory system, and multiple mechanisms likely exist to generate them. Under specific conditions, offset responses can be created by cochlear mechanics (Box 1). In this Review, however, we focus primarily on neural mechanisms driving offset responses found in the central auditory system. In each part of the auditory system described below, researchers have successfully distinguished offset responses from delayed onset responses, by demonstrating that offset responses remain time-locked to sound termination when sound duration is varied [3,5,25,41-44].

\section{Cochlear Nucleus}

The deep layer of the dorsal cochlear nucleus (DCN) is the earliest auditory brain structure in which excitatory offset responses occur [45]. Offset firing has been observed in Type III and IV neurons [5,46], which are likely to be principal or giant cells [47] projecting to the contralateral inferior colliculus [48]. Type III neurons have V-shaped tuning curves flanked by inhibitory sidebands, whereas type IV neurons have more complex tuning, with wide-band inhibitory input especially at higher sound intensities $[5,49]$. Inhibitory inputs to the DCN arrive with the delay of at least one additional synapse relative to auditory nerve inputs, and are thought to contribute to monaural echo suppression and generation of the precedence effect [50] as well as to generation of offset responses.

For near-threshold tones at characteristic frequency (CF), Type IV neurons typically produce a transient response to tone onset [5]. Offset responses to tonal stimuli in DCN cells occur following sound frequencies and intensities that elicit inhibition of the neuron's response during the tone, with intensity thresholds that can be as low as 20 dB SPL [49]. In vivo intracellular recordings indicate that DCN offset responses might be generated by a post-inhibitory rebound mechanism [51], and current-clamp recordings show that fusiform/giant cells may generate one or two action potentials (APs) after hyperpolarizing current injections [46]. However, the mechanisms 
underlying the robust offset responses that have been observed in the DCN of anaesthetized bats [45] and decerebrate cats [5] remain to be confirmed.

\section{Superior Olivary Complex}

Cells of the superior paraolivary nucleus (SPN) in the superior olivary complex (SOC) generate pronounced offset responses following tone cessation, with a latency of $2-7 \mathrm{~ms}[9,52]$, as well as responses to gaps as short as 3ms [15, 53]. Neurons with very similar response properties were first observed within the bat medial superior olive (MSO) [2,54], and seem likely to represent the same type of cell as the offset-responsive neurons that are concentrated in SPN of other species. Offset responses are observed in nearly all SPN cells, whereas onset responses are observed in fewer than 10\% [55]; moreover, offset responses are more robust than onset responses to changes in stimulus bandwidth (Gomez-Alvarez et al. 2018). SPN neurons are under constant inhibitory constraint from spontaneous activity in the medial nucleus of the trapezoid body (MNTB), and are strongly inhibited by MNTB activity during sound stimulation $[2,56,57]$. When MNTB neurons are briefly silenced by post-stimulus suppression, SPN neurons generate APs via post-inhibitory rebound $[56]$.

Post-inhibitory rebound mechanisms are distinct from other related cellular phenomena such as post-hyperpolarization events or post-inhibitory facilitation (Figure 2). Briefly, post-hyperpolarization events occur without synaptic inputs, solely based on the interaction of intrinsic ionic conductances (for example, in rhythm generation [58,59]). In post-inhibitory facilitation, by contrast, both excitatory and inhibitory synaptic inputs are required to generate the response; inhibition enhances recovery of voltage-gated sodium and calcium channels from inactivation to boost subsequent excitation. This mechanism is thought to increase temporal precision of excitatory input in the auditory brainstem, especially in binaural coincidence detectors $[60,61]$, and has also been suggested as a potential mechanism for creating sound-offset responses [57]. Note that in post-inhibitory facilitation, the inhibitory 
input alone does not trigger an offset response.

Post-inhibitory rebound mechanisms differ from both post-hyperpolarization events and post-inhibitory facilitation in being driven by inhibitory input alone. For example, in the SPN, neurons strongly express the neuronal potassium-chloride co-transporter KCC2, which maintains a low internal chloride concentration $(<5 \mathrm{mM})$ [62]. When chloride channels open following activation of glycine or gamma amino butyric acid (GABA) receptors, chloride enters the neurons driven by a strong chemical driving force towards the chloride equilibrium potential $\left(E_{\mathrm{cl}}\right)$ of about $-100 \mathrm{mV}[53,56,62]$. Such large hyperpolarizations activate hyperpolarization-activated cyclic nucleotide-modulated currents $\left(\mathrm{I}_{\mathrm{H}}\right) \cdot \mathrm{I}_{\mathrm{H}}$ currents are mediated by a mixture of $\mathrm{Na}^{+}, \mathrm{K}^{+}$ and $\mathrm{Ca}^{2+}$ ions, rendering the reversal potential of $\mathrm{I}_{\mathrm{H}}$ a mixture of the equilibrium potentials of each of the ions involved. In SPN neurons the reversal potential of $I_{H}$ is positive to the resting membrane potential, so as the membrane voltage changes from near $\mathrm{E}_{\mathrm{Cl}}$ towards the resting membrane potential of about $-55 \mathrm{mV}$, low-voltage activated T-type calcium currents are activated around -80mV [56,63]. Activation of T-type currents then drives the membrane potential towards $+125 \mathrm{mV}\left(\mathrm{E}_{\mathrm{Ca}}{ }^{2+}\right)$, activating sodium channels at around $-30 \mathrm{mV}$. Since T-type calcium currents also inactivate in a voltage-dependent manner, calcium influx is only possible during a small voltage window from -80 to $-50 \mathrm{mV}$ [56]. The resulting calcium transient generates a brief depolarizing potential that carries a burst of 3-4 sodium-mediated APs.

Thus, SPN neurons combine three properties which together allow them to exhibit rebound firing in response to physiological input. First, acoustically or electrically evoked synaptic inhibition causes SPN neurons to reach membrane potentials as negative as $-100 \mathrm{mV}$. Second, this hyperpolarization activates large $I_{H}$ and T-type conductances. Third, the resting membrane potential of SPN neurons is unusually depolarized compared to that of neurons in the surrounding auditory nuclei, providing a large driving force for inwardly rectifying conductances.

At the biophysical and cellular level, post-inhibitory rebound in the SPN is currently the best understood mechanism of offset response generation in the auditory system. 
At the circuit and systems level, however, many questions remain. The SPN is suggested to contain GABAergic neurons $[64,65]$ although glycinergic neurons have also been reported [65]. Downstream targets of the SPN include the IC and the MGB [66], but the functional influence of SPN projections has only recently begun to be explored [70]. Therefore, the precise role of the SPN in central auditory processing still needs to be clarified.

\section{Inferior Colliculus}

The inferior colliculus (IC) receives inputs from many brainstem nuclei including the DCN and SPN. IC neurons exhibit a mix of onset, sustained, inhibited and offset responses to tones [42]. Reported prevalence of excitatory offset responses in IC ranges from $9 \%$ [42] to $70 \%$ [38]. The latency of offset responses is longer ( $\geq 9 \mathrm{~ms})$ in IC than in SPN or CN $[9,42]$, and the number of APs in IC offset responses varies with sound duration [41] (Box 2). Frequency tuning of onset and offset responses tends to overlap in IC neurons with either transient onset-offset or onset-sustained-offset response profiles $[42,67]$.

The majority of offset responses in the IC appear to arise via inheritance from upstream generators. Example offset responses recorded in bat IC were unaffected by local blockade of inhibition [68], suggesting that the responses were driven by excitatory input to the IC. Intracellular recordings in vivo demonstrated that offset responses in the IC can be generated in both principal bipolar cells and inhibitory multipolar cells via a post-inhibitory rebound mechanism; however this was observed in only 2/11 neurons, whereas 9/11 fired in response to a purely excitatory input arriving at the end of the tone [42]. These excitatory inputs could arise from the contralateral DCN where offset responses are found in neurons likely to be principal neurons, projecting to the IC [49].

However, the IC is also noted for its complex inhibition-driven physiology. Post-inhibitory facilitation in IC creates a temporal window during which subsequent excitation may produce bursts of APs. This mechanism is thought to generate tuning 
to sound durations between one and several hundred milliseconds depending on the species [41,42]. Additionally, combination-sensitive neurons in the IC (i.e. neurons that fire in response to simultaneous presentation of two spectrally distinct tones) may be activated following summation of the post-inhibitory rebounds generated in response to the two tones, while presentation of just one tone is insufficient to produce firing [69]. Xie et al. [67] showed that, generally, higher frequency tones evoke inhibitory post-synaptic potentials (IPSPs) at tone onset and lower frequency tones evoke IPSPs at offset. A combination presenting first the low-CF tone and then the high-CF tone allows coincidence of the IPSPs, generating sufficient rebound depolarization to produce APs solely via the combination of two glycinergic inputs [69].

A recent study in mice found offset-responses in 69.7\% (85/122) of IC neurons, more commonly in cells with frequency-intensity response areas exhibiting inhibitory sidebands [38]. Of the offset-responding cells, more than half (54\%) showed facilitation of the offset response when pup communication calls or stimuli with multiple frequency components were presented rather than pure tones [38]. These results suggest that the prevalence of offset responses in the IC may depend on the spectral complexity and/or behavioral relevance of the stimulus, and are consistent with the idea that duration tuning, combination sensitivity, and offset responses in the IC may share some common underlying neural mechanisms.

In the first reported attempt to manipulate offset response pathways, Felix et al. [70] silenced SPN pharmacologically and recorded in IC. They observed no significant effect of this manipulation on the number of offset spikes generated in IC. However, they found that SPN inactivation disrupted entrainment of IC spiking to some sinusoidally amplitude-modulated tone stimuli, and also increased gap-detection thresholds in IC cells with sustained responses. These results suggest that well-timed inhibition from SPN, in combination with other excitatory and inhibitory inputs, helps IC neurons follow rapidly modulated signals.

In summary, IC offset responses to tones may largely be driven by excitatory inputs from the DCN, with inhibition from the nuclei of the lateral lemniscus and SPN acting 
to shape more complex response types [41]. Summation of inhibition in response to presentation of multiple tones may additionally generate de novo offset responses to frequency combinations important in behavior [38,69]. However, the cellular and/or network origins of offset responses in the IC remain to be fully elucidated.

\section{Medial Geniculate Body}

The medial geniculate body of the thalamus (MGB) receives excitatory and inhibitory inputs from the IC and from brainstem nuclei including the DCN and SPN [66]. The MGB is divided into ventral (MGv), dorsal (MGd), and medial (MGm) subdivisions, and also includes other subregions such as the suprageniculate MGB (MGs). MGv is part of the lemniscal (primarily auditory) pathway. Offset responses have been found in MGv and MGs but also in non-lemniscal (multisensory) regions [10,25,43,44], and are concentrated in spatially segregated sheets around the borders of all areas of the MGB [44]. In MGv, offset responses occur most often in neurons that also exhibit onset responses $[10,25]$; offset-only response types are found primarily in the non-lemniscal and border regions. Mean latencies of offset responses range from around 20-25ms in MGv [10,25] to $\geq 30 \mathrm{~ms}$ in other regions (MGd, MGs) [10]. Thresholds for sound-evoked activity are higher for offset than onset responses in MGB [10]. Characteristic frequencies are also typically higher for offset than onset responses, leading to onset/offset tuning asymmetry in neurons with both responses $[10,25]$.

Offset responses in MGB may be caused by excitation arriving after tone offset, which could be amplified through post-inhibitory facilitation following cessation of sustained inhibition during a tone. Intracellular recordings from MGB neurons in vivo demonstrate sound-offset responses in the form of 1-3 excitatory postsynaptic potentials or APs without inhibitory input [43]. Corticofugal modulation may boost the offset response $[71,72]$, potentially via post-inhibitory facilitation. Notably, although in vitro injection of hyperpolarizing current into MGB neurons can generate post-hyperpolarization offset responses [73-76], there is currently no in vivo evidence (to our knowledge) for generation of offset responses in the MGB through 
post-inhibitory rebound alone.

A recent study of MGB in the ectopic BXSB/MpJ-Yaa mouse model of developmental disorder found a reduction in the proportion of neurons with offset responses in the MGv, but no apparent abnormalities in MGv onset responses to noise bursts, click trains or tones [25]. For MGv neurons with both onset and offset responses to tones, onset/offset asymmetry in frequency tuning was reduced in affected animals, and this reduction appeared to arise from a change in offset response tuning alone. Moreover, observed abnormalities in MGv population responses to gap-in-noise stimuli could be reproduced in a phenomenological model incorporating intensity gain control and dissociable offset- and onset-sensitive channels, simply by varying the weighting of the offset-sensitive channel. These findings suggest that offset responses in MGv are primarily driven by dedicated brainstem generators, rather than arising from more general neural mechanisms that would be expected to affect both onset and offset responses if disrupted.

\section{Auditory Cortex}

Auditory cortex $(\mathrm{AC})$ receives inputs from MGB and is the origin of descending inputs to many structures upstream in the auditory pathway $[77,78]$. Studies performed in awake animals typically report offset responses in 30-70\% of neurons in primary AC depending on the species (monkey: [79]; rat: [80]; cat: [81]; mouse: [82]); additionally, Tian et al. [83] reported that $90 \%$ of cells in auditory cortex of awake and behaving monkeys generate offset responses to bandpass noise. Cortical offset responses usually occur in neurons that also exhibit onset responses [84], and have been observed in interneurons as well as presumed pyramidal cells [82], and not only in primary AC but also in secondary auditory cortical fields (monkey: [79,85,86]; rat: [80]).

In contrast to offset responses further upstream in the central auditory pathway, cortical offset responses appear to be independent of prior inhibition [81]. In vivo whole-cell recordings in A1 show that inhibitory synaptic potentials do not last for the 
full stimulus duration and thus cannot generate either post-inhibitory rebound or post-inhibitory facilitation [3]. Offset responses in cortex instead seem to be driven by excitatory post-synaptic potentials arriving after the tone, which are distinct from those mediating the onset response [3]. The latencies of onset and offset responses are generally reported to be $10-30 \mathrm{~ms}$ in cortex, with offset response latency (relative to tone offset) slightly slower than onset response latency $[81,87,88]$. Response duration is typically longer for offset than onset responses in cortex [88]; indeed, offset responses lasting hundreds of milliseconds following sound cessation have been reported in cat and ferret [89,90]. Long-lasting sound-offset responses have also been observed in the mouse auditory cortex following very long (7s) tones [91]; however, this form of offset response does not occur even following 1s tones, and seems to be generated through general synaptic plasticity mechanisms rather than the offset-specific mechanisms that are our primary focus here.

In cortex, there is little evidence for clustering of offset responses. Most single-unit recording studies have not reported spatial segregation of offset-responsive neurons, instead indicating that offset responses occur throughout the $A C$, especially in neurons that also produce transient onset responses. However, cortical surface recording and intrinsic signal optical imaging studies have suggested that offset-sensitive neurons could be concentrated between or at the edges of strongly tonotopic cortical regions [80,91]. Laminar differences in offset response incidence have also been observed: Volkov \& Galazjuk [84] found that phasic neurons, which are more likely to exhibit offset firing than sustained neurons, tended to be present in layer $2 / 3$, whereas sustained neurons, which were less likely to generate offset responses, tended to be located in layers 5 and 6 .

As in the MGB, neurons with both onset and offset responses often show onset/offset asymmetry in frequency tuning $[3,81,85,92]$; asymmetry in binaural tuning has also been reported $[85,88]$. These asymmetries may provide key insights into how central auditory processing works, as discussed below.

Overall, the existing data on offset responses in the central auditory pathway suggest that offset responses are primarily generated in the DCN and SPN; then combined 
with other sound cues in the IC; and lastly used in the MGB and cortex as a substrate for building flexible representations of behaviorally important sounds (Figure 3, Key Figure).

\section{Asymmetries in Onset and Offset Response Properties}

Onset and offset responses, even in the same neuron, often have different response properties, but the origins and functional significance of these asymmetries are still poorly understood. The most obvious asymmetry is in onset and offset response strength $[3,25,42,44,81,84,85,88]$. As previously discussed (see "Computational Significance of Sound Offsets in Auditory Processing"), weaker offset compared to onset responses might reflect evolutionary adaptation to the characteristics of natural sounds.

Onset/offset asymmetry in frequency tuning is another prominent feature of central auditory responses, particularly in the thalamus and cortex. Some studies have found that preferred frequencies (both CF and BF) are higher on average for offset than onset responses in neurons that generate both types of response (e.g., [3,10,25]). Other studies have found that offset responses are equally likely to be tuned to higher or lower frequencies than onset responses [81,85,92]. In mouse auditory cortex, Sollini et al. [26] recently showed that the sign of onset/offset asymmetry depends on the frequency tuning of the onset response, and correlates with neuronal selectivity for direction of frequency-modulated (FM) tone sweeps. Results were consistent with a model in which FM direction selectivity arises from temporal coincidence of offset and onset responses; for example, when the offset response is tuned to higher tone frequencies than the onset response, a downward FM sweep of appropriate speed generates temporally aligned offset and onset depolarizations that summate to drive stronger firing than would be evoked by upward sweeps. Thus, asymmetry in frequency tuning of onset and offset responses may contribute to neural encoding of FM sweep direction.

Asymmetry in spatial tuning of onset and offset responses has also been reported. In 
auditory cortex of anaesthetized ferret, Hartley et al. [88] found anti-correlated onset and offset response tuning for both interaural level difference and interaural time difference cues. Spatial tuning of onset and offset responses was reportedly more consistent for auditory cortical neurons in awake macaque [85], although variability in onset/offset asymmetry across neurons was still high. Importantly, binaural cues are initially detected in superior olivary nuclei other than the SPN, and relayed to the inferior colliculus. The presence of differences in binaural tuning between onset and offset responses therefore supports the idea that offset responses could be generated de novo in the IC. Furthermore, just as asymmetry in frequency tuning of onset and offset responses may shape neuronal selectivity for FM sweeps, asymmetry in spatial tuning could confer selectivity for auditory motion.

The origins of observed asymmetries in onset and offset response properties remain unclear. One possibility is that onset/offset asymmetry reflects fundamental differences in response properties between onset- or offset-sensitive auditory brainstem neurons providing input to higher auditory brain areas. A second possibility is that onset/offset asymmetry reflects emergent differences between ascending onset- and offset-sensitive pathways, perhaps arising from stimulus-driven plasticity or adaptation. For example, Sollini et al. [26] showed that with frequency-tuned onset- and offset-selective inputs, an output neuron can develop onset/offset asymmetry in frequency tuning through simple Hebbian plasticity, essentially because tone onsets and offsets cannot occur simultaneously in the same frequency channel. Time-dependent circuit or network mechanisms, such as those recently proposed to explain emergent inhibitory sidebands in cortical frequency tuning curves [93], might also introduce asymmetries between onset and offset responses. For example, divergent spatial tuning in onset and offset responses might arise from effects of network suppression on "push-pull" integration of excitatory and inhibitory inputs shaping binaural selectivity [94]. Thus, it seems possible that asymmetry in onset and offset response properties might be a consequence of a much more general rule: that neural representations change as a function of time. 


\section{Concluding Remarks and Future Perspectives}

Like offset responses in other sensory systems [19,20], sound-offset responses may improve metabolic efficiency of stimulus encoding and simplify biophysical implementation of behaviorally relevant nonlinear computations. Sound-offset responses are also likely to be essential for perceptual grouping, duration discrimination, gap detection, and consonant identification. However, many questions about sound-offset responses in the auditory system remain unanswered (see Outstanding Questions).

Neurons with sound-offset responses are found throughout the auditory system and are concentrated in particular nuclei of the brainstem and subregions of the thalamus, suggesting the existence of an "offset pathway" in the auditory brain. The neural mechanisms of sound-offset response generation are well-understood for the SPN in the brainstem, but remain to be fully elucidated for the DCN, IC, MGB and cortex. Understanding the cellular and circuit origins of sound-offset responses in the auditory brain may be key not only to cracking the neural code for temporally discontinuous sounds such as speech, but also to advancing treatment of auditory temporal processing deficits in aging and disease.

\section{Acknowledgements}

We thank Benedikt Grothe, Lutz Wiegrebe, Jonathan Ashmore, Torsten Marquardt, Maria Chait, Joseph Sollini, Jane Mattley and Fatima Ali for helpful discussions and comments on manuscript drafts. We also gratefully acknowledge the support of the Medical Research Council (MR/P006221/1), the Royal Society (IE140709), Action on Hearing Loss (F44, F61, G77), the German Research Council (DFG KO2207/3-1) and the National Institute for Health Research University College London Hospitals Biomedical Research Centre. 


\section{BOX 1: Sound-Offset Responses in the Cochlea and Auditory Nerve}

Sound-offset responses have occasionally been reported in the cochlea and auditory nerve [95-99], although there is debate about whether these responses should be considered physiologically relevant phenomena or artefacts of particular sound stimuli. Lewis and Henry [99] showed theoretically that onset and offset responses to a tone burst can appear in the time-domain output of a simple linear bandpass filter provided both that the frequency of the tone is well outside the pass band and that the slope of the band edge is sufficiently steep. They argued that the steep high-frequency rolloff in cochlear frequency tuning means these criteria may be fulfilled in the cochlea depending on the stimulus frequency. This idea is more easily understood in the frequency domain; fluctuations in tone intensity such as onset and offset ramps contain a wider range of sound frequencies than the steady part of the tone, and under certain conditions this "spectral splatter" can drive onset and offset responses in auditory nerve fibres that do not respond to the tone frequency. Sound-offset responses in the cochlea and auditory nerve could also arise from active cochlear mechanisms amplifying residual vibrational energy in the basilar membrane following sound stimulation [100]. This mechanism has been proposed to explain offset responses in the bat auditory nerve [95]. Thus, it is theoretically possible that some central auditory offset responses might be inherited from the peripheral auditory system.

However, the reported characteristics of offset responses in the auditory nerve differ in important ways from the central auditory offset responses that are the focus of this Review. First, sound-offset responses in the auditory nerve are most commonly evoked by high-intensity, high-frequency tones, in neurons that also produce sustained primary-like responses without offset firing for low-intensity, lower frequency tones $[96,98,99]$. Second, offset responses in the auditory nerve only occur for tones that also produce onset responses in the same neurons $[95,96,98]$. Third, offset responses in the auditory nerve can be elicited even following very short (e.g., 2-8ms) sounds [96]. In contrast, in the central auditory system, sound-offset responses can manifest as the dominant response pattern of a neuron even for low-intensity, low-frequency tones; may occur even in the absence of an onset 
response; and are often evoked only following sounds lasting many tens (or even hundreds) of milliseconds. 


\section{BOX 2: Sound Offsets and Duration Encoding}

Sound-evoked offsets provide an essential cue for sound duration encoding. Sound duration changes perception of loudness [101] and is important for detection of phonetic boundaries during vocal communication [1]. Neural mechanisms of duration encoding have been extensively studied in bats [41,102], vocalizing anurans $[103,104]$, and other vertebrates including humans [105-109]. In humans as well as other mammals, differences in duration between two sounds can be perceived only when the difference is at least $10-20 \mathrm{~ms}[110,111]$. Although this temporal precision is almost two orders of magnitude slower than is achieved in sound localization pathways, sound duration encoding seems to be well adapted to the duration of conspecific communication sounds [112,113].

In humans, duration discrimination is significantly better for filled intervals (continuous sounds) compared to empty intervals (silent time between two clicks) [114]. This discrepancy is most likely due to different mechanisms underlying the duration encoding of the two types of stimuli. While continuous sounds evoke an onset response at the beginning and an offset response at the end, two brief clicks separated by the same duration as the length of the continuous sound will evoke two separate onset responses and no offset response [115]. Latencies of brainstem offset responses depend on activation kinetics of $\mathrm{I}_{\mathrm{H}}$ [116] and thus are inversely proportional to the duration of the prior sound $[56,117]$. If a sound is of sufficient duration $(T)$ to allow for an offset response, the just-noticeable difference between two sounds of different durations $(\Delta T)$ should be smaller. Indeed, $\Delta T$ decreases with increasing sound duration $(T)$, so that the Weber fraction $(\Delta T / T)$ is smaller for longer sounds [118].

Duration-sensitive neurons act as temporal filters and produce responses characterized as shortpass (e.g., maximal response to tones of 20ms or less), bandpass (maximal response at a specific duration, e.g. 50ms, with fewer APs in response to shorter or longer stimuli) or longpass (maximal response to a stimulus of long duration, e.g. 70ms, and increasing the duration of the sound does not increase 
the response magnitude further) [116,119,120]. Currently proposed mechanisms of duration tuning include a coincidence model in which slightly delayed onset excitation coincides with short-latency offset excitation. Another version of such a coincidence model requires delayed subthreshold onset excitation which summates with rebound from short-latency offset inhibition $[119,120]$. The main idea behind both of these models is that the onset response is delayed to coincide with an offset response. Delaying of the onset response could be achieved in many possible ways: for example, by varying axon diameter and myelin thickness, by deploying A-currents, or by expressing slower versus faster AMPA receptors. However, even a combination of these mechanisms would delay neural signals by only a few tens of milliseconds. For longer sound durations, an anti-coincidence model has been proposed in which sustained stimulus-driven but rapidly adapting inhibition initially counteracts but then is overcome by sustained and less rapidly adapting excitation [121]. Duration-dependent disinhibition might then be further amplified by rebound from inhibition at stimulus offset. 


\section{Box 3: Sound-Offset Responses in Aging and Disease}

Standard clinical hearing tests assess sensitivity to sound onsets, not offsets. If specific abnormalities in sound-offset responses occur, they would be expected to manifest not as problems with tone detection thresholds but as impairments in temporal processing and ability to perceive rapidly varying signals. It is not yet clear how human performance in tests of auditory temporal processing, such as gap-in-noise detection or speech discrimination, might depend upon auditory brain sensitivity to sound offsets rather than onsets. However, animal studies suggest that specific impairments in sound-offset sensitivity can occur naturally [25] and might produce gap-detection deficits $[25,70]$.

Gap-detection duration thresholds are higher in older than younger adults, even when hearing thresholds are normal $[122,123]$. Older adults also have more difficulty with speech discrimination in noise [124], and these difficulties are well-simulated as arising from increased temporal jitter in the auditory system [125]. Notably, offset-related components of auditory evoked responses to gap-in-noise stimuli and speech sounds are significantly weaker and more delayed in older than younger adults [126,127], although onset-related components are also affected by aging. Abnormalities in gap detection and brain responses to stimulus transients, including sound offsets, have also been reported in subjects with developmental or neurological disorders $[128,129]$ and in animal models of aging and disease $[1,11]$.

A likely cause of gap-detection deficits and other auditory temporal processing difficulties is alteration in excitatory and inhibitory synaptic transmission, particularly weakening of inhibition $[1,11,130]$. Notably, in mouse models of auditory neuropathy and tinnitus, emergence of auditory temporal processing deficits has been linked to abnormalities in excitation-inhibition balance in the auditory midbrain and cortex $[131,132]$. The best-understood mechanism of sound-offset response generation post-inhibitory rebound in the SPN - relies upon strong inhibitory synaptic input [56], and other proposed mechanisms for de novo generation of sound-offset responses depend critically upon interaction between excitatory and inhibitory inputs (Figure 2). Therefore, sound-offset responses may be particularly sensitive to disruptions of 
excitation-inhibition balance in aging and disease. 


\section{Glossary}

Between-channel gap-detection threshold: Duration threshold for detection of silent gaps when there is a spectral disparity between the sounds leading and trailing the gap; typically $30 \mathrm{~ms}$.

Characteristic frequency (CF): The sound frequency at which a neuron responds to the lowest sound intensity (i.e., the frequency to which it is most sensitive).

Empty interval: Time interval between two clicks.

Filled interval: Time interval between the start and the end of a continuous sound.

Post-hyperpolarization events: Action potential firing which occurs entirely without synaptic inputs due to the interaction of intrinsic ionic conductances following hyperpolarization of a neuron (e.g., rhythm generation in thalamic or respiratory neural networks).

Post-inhibitory facilitation: Mechanism in which delayed subthreshold excitation is boosted by preceding inhibition through enhanced recovery of voltage-gated sodium channels from inactivation. Both excitatory and inhibitory synaptic inputs are required to generate a response.

Post-inhibitory rebound: Action potential firing initiated by an inhibitory synaptic input, in which action potentials are produced at the end of the hyperpolarizing inhibitory input without any need for additional excitatory input. Note that post-inhibitory rebound can be induced experimentally in most neurons with sufficiently extreme hyperpolarization; here we refer instead to the natural phenomenon induced by physiological levels of inhibitory synaptic input.

Post-stimulus suppression: Suppression of action potential firing below spontaneous rate following a sound offset, for example due to delay in recovery from stimulus-driven adaptation in sensory transduction mechanisms.

Sound offset: A sound termination, usually involving a drop in sound intensity. 
Sound-offset response: A transient increase in the activity of a neuron, time-locked to a sound offset. Note that transient decreases in neural activity time-locked to sound offset can arise simply from neural mechanisms implementing adaptation or high-pass filtering. We define sound-offset responses as involving transient increases in firing because these responses are more likely to arise from dedicated offset-sensitive cellular or network mechanisms.

Within-channel gap-detection threshold: Duration threshold for detection of silent gaps in an otherwise continuous sound; typically $2-3 \mathrm{~ms}$. 


\section{Figure Legends}

Figure 1. Computational significance and perceptual characteristics of sound offsets.

A. Neural representation of sound transients may be more metabolically efficient with dual onset/offset pathways than with a single pathway. Left, Diagram representing stimulus with varying sound level. Middle, To represent both increases and decreases in sound level, transient detectors in a single neural pathway would need to maintain high spontaneous firing rates. Right, With a dual pathway of separate onset and offset detectors, the same signal can be represented by neural pathways with very low spontaneous rates.

B. Voice-onset times - gaps between release of a vocal tract closure and onset of vocal fold vibration - are cues for consonant discrimination. Left and middle, Spectrograms of a male speaker saying the phonemes Idal and Ital. The voice-onset time (VOT) is shorter for Idal than Ital. Right, English speakers show categorical perception of VOT when this parameter is varied systematically along the |dal-|tal continuum. Adapted from Sharma and Dorman [28].

C. In natural sounds, offsets are often less abrupt than onsets. Left, Impact sounds like a plate breaking typically have a fast onset and a more slowly decaying offset. Middle, Reverberation further prolongs the sound offset, as in the example of a door slamming in a reverberant room. Right, Offsets can be more pronounced within communication sounds such as speech. In this example, a female speaker is saying the phrase "encoding endings".

Figure 2. Cellular and synaptic mechanisms underlying sound-offset responses. A) Post-inhibitory rebound firing: Inhibitory input(top row, blue trace) during the sound (solid black bar) hyperpolarizes the membrane to $\mathrm{E}_{\mathrm{Cl}^{-}}$(-80 to $-90 \mathrm{mV}$, turquoise shaded area). The repetitive peaks in the inhibitory input represent multiple synaptic potentials in response to the stimulus. The hyperpolarization during the sound allows T-type calcium channels to recover from inactivation; T-type calcium 
channels usually activate and inactivate around resting potential (-50 to $-60 \mathrm{mV})$. After the sound ends (light gray shaded area), the membrane potential depolarizes towards resting potential. The depolarized resting potential strongly activates the newly available T-type calcium channels, generating a large calcium transient (dark gray area under curve). Thus, despite the absence of excitatory input (middle row), the calcium transient depolarizes the membrane past threshold $(-40 \mathrm{mV})$, triggering a burst of action potentials (bottom row, black trace).

B) Post-inhibitory facilitation: In this case, rebound depolarization is insufficient to trigger an action potential and must be combined with excitation to generate spiking. As in post-inhibitory rebound firing, inhibition during the sound (top row) generates persistent hyperpolarization, allowing T-type calcium currents to recover from inactivation. When the sound stops, a calcium transient is generated (dark gray area under curve). Given the more hyperpolarized resting potential of this cell (compared to cells which exhibit post-inhibitory rebound), the transient is insufficient to generate offset firing. The cell also receives excitatory synaptic input during the sound (middle row). In isolation, this excitatory input is insufficient to trigger an action potential, but in combination with the calcium transient produced by release from inhibition at the end of the tone, the delayed excitatory synaptic input causes membrane potential to cross the spiking threshold, generating a rebound action potential (bottom row, black trace).

C) Inherited offset response: Excitatory post-synaptic potentials (middle row, red trace) capable of triggering action potentials (bottom row, black trace) are received from offset-sensitive neurons in the upstream pathway, allowing offset responses to be transmitted to higher centers. This mechanism does not require inhibitory inputs or rebound depolarization. 


\section{Figure 3. Key Figure: An "offset pathway" in the auditory system?}

A. Sagittal schematic of mouse brain. Afferent signals from the cochlea may generate offset responses in the cochlear nucleus (CN) and superior olivary complex (SOC). Auditory signals are relayed to the inferior colliculus (IC), medial geniculate body of the thalamus (MGB), and ultimately to the auditory cortex (AC).

B. Coronal schematics of mouse auditory brainstem and forebrain. Red lines represent excitatory signals, blue lines inhibitory ones. Bottom, Brainstem pathways. Input from auditory nerve (dotted lines) generates offset responses (solid lines) in dorsal cochlear nucleus (DCN) type III \& IV cells $[5,46]$. Sustained activity of bushy cells of the ventral cochlear nucleus (VCN) drives the contralateral medial nucleus of the trapezoid body (MNTB) which in turn provides powerful inhibition to SOC nuclei including the lateral superior olive (LSO) and superior paraolivary nucleus (SPN). Post-inhibitory rebound generates offset responses in SPN following cessation of sustained inhibition from the MNTB [56]. Cells in the inferior colliculus (IC) may fire at tone offset, driven by excitatory post-synaptic potentials (EPSPs) arriving after a tone, presumably from DCN [42]. IC receives inhibitory input from the from nuclei of the lateral lemniscus (not shown) and the SPN (blue input line). Inhibitory input may summate to generate offset responses to behaviorally relevant sounds (e.g. conspecific vocalizations or sounds of a particular length). Cells from the central and external cortex of IC (CIC and $\mathrm{ECIC})$ project to the medial geniculate body of the thalamus (MGB).

Top, Forebrain pathways. The MGB is divided into dorsal (MGd), medial (MGm), ventral (MGv) and suprageniculate (MGs) subdivisions. Offset responses tend to be found in sheets surrounding MGB subdivisions [44]. Offset responses are generated by EPSPs arriving after the tone, presumed to be from IC [43]. Input from other brain areas (e.g., SPN) might contribute IPSPS arriving after the tone, producing post-stimulus suppression [42]. MGB projects to auditory cortex (AC). Offset responses in cortex are generated by EPSPs arriving after the tone, from a distinct 
population of synapses to those mediating the onset response [3].

C. Coronal sections from the mouse brain showing (from top): AC [133], MGB [25], IC, SPN and DCN (unpublished images, Kopp-Scheinpflug lab).

D. Peri-stimulus time histograms of neural responses to tones in different brain regions (the duration of the tone varies between the examples, from 60 to 200ms). From top: mouse AC [3], guinea pig MGB [44], mouse IC [38], gerbil SPN [9], and cat DCN [5]. Neurons often produce both a transient onset response (orange, in top panel) and a transient offset response (blue, in top panel), with the offset response occurring between $5 \mathrm{~ms}$ and $50 \mathrm{~ms}$ after tone offset depending on position in the pathway. Neurons with a transient offset response in the absence of other responses to tones have been observed primarily in SPN and in some regions of MGB. 


\section{References}

1. Eggermont, J.J. (2015) Auditory Temporal Processing and its Disorders, Oxford University Press.

2. Grothe, B. (1994) Interaction of excitation and inhibition in processing of pure tone and amplitude-modulated stimuli in the medial superior olive of the mustached bat. J Neurophysiol 71 (2), 706-21.

3. Scholl, B. et al. (2010) Nonoverlapping sets of synapses drive on responses and off responses in auditory cortex. Neuron 65 (3), 412-21.

4. Phillips, D.P. et al. (2002) Central auditory onset responses, and temporal asymmetries in auditory perception. Hear Res 167 (1-2), 192-205.

5. Young, E.D. and Brownell, W.E. (1976) Responses to tones and noise of single cells in dorsal cochlear nucleus of unanesthetized cats. J Neurophysiol 39 (2), 282-300.

6. Cavaco, S. and Lewicki, M.S. (2007) Statistical modeling of intrinsic structures in impacts sounds. J Acoust Soc Am 121 (6), 3558-68.

7. Deneux, T. et al. (2016) Temporal asymmetries in auditory coding and perception reflect multi-layered nonlinearities. Nat Commun 7, 12682.

8. Sohoglu, E. and Chait, M. (2016) Neural dynamics of change detection in crowded acoustic scenes. Neuroimage 126, 164-72.

9. Dehmel, S. et al. (2002) Electrophysiological characterization of the superior paraolivary nucleus in the Mongolian gerbil. Hear Res 172 (1-2), 18-36.

10. He, J. (2002) OFF responses in the auditory thalamus of the guinea pig. J Neurophysiol 88 (5), 2377-86.

11. Felix, R.A., 2nd et al. (2018) Subcortical pathways: Towards a better understanding of auditory disorders. Hear Res 362, 48-60.

12. Grothe, B. et al. (1997) Medial superior olive in the free-tailed bat: response to pure tones and amplitude-modulated tones. J Neurophysiol 77 (3), 1553-65.

13. Burger, R.M. and Pollak, G.D. (1998) Analysis of the role of inhibition in shaping responses to sinusoidally amplitude-modulated signals in the inferior colliculus. J Neurophysiol 80 (4), 1686-701.

14. Kiang, N.Y. et al. (1965) Stimulus coding in the cochlear nucleus. Trans Am Otol Soc 53, 35-58.

15. Kadner, A. and Berrebi, A.S. (2008) Encoding of temporal features of auditory stimuli in the 
medial nucleus of the trapezoid body and superior paraolivary nucleus of the rat. Neuroscience 151 (3), 868-87.

16. Zilany, M.S. et al. (2009) A phenomenological model of the synapse between the inner hair cell and auditory nerve: long-term adaptation with power-law dynamics. J Acoust Soc Am $126(5), 2390-412$.

17. Eichner, H. et al. (2011) Internal structure of the fly elementary motion detector. Neuron 70 (6), 1155-64.

18. Chalasani, S.H. et al. (2007) Dissecting a circuit for olfactory behaviour in Caenorhabditis elegans. Nature 450 (7166), 63-70.

19. Westheimer, G. (2007) The ON-OFF dichotomy in visual processing: from receptors to perception. Prog Retin Eye Res 26 (6), 636-48.

20. Gjorgjieva, J. et al. (2014) Benefits of pathway splitting in sensory coding. J Neurosci 34 (36), 12127-44.

21. Xu, N. et al. (2014) The function of offset neurons in auditory information processing. Translational Neuroscience 5, 275-285.

22. Atencio, C.A. et al. (2008) Cooperative nonlinearities in auditory cortical neurons. Neuron $58(6), 956-66$.

23. Williamson, R.S. et al. (2016) Input-Specific Gain Modulation by Local Sensory Context Shapes Cortical and Thalamic Responses to Complex Sounds. Neuron 91 (2), 467-81.

24. Loebel, A. et al. (2007) Processing of sounds by population spikes in a model of primary auditory cortex. Front Neurosci 1 (1), 197-209.

25. Anderson, L.A. and Linden, J.F. (2016) Mind the Gap: Two Dissociable Mechanisms of Temporal Processing in the Auditory System. J Neurosci 36 (6), 1977-95.

26. Sollini, J. et al. (2018) ON-OFF receptive fields in auditory cortex diverge during development and contribute to directional sweep selectivity. Nat Commun 9 (1), 2084.

27. Bregman, A.S. (1990) Auditory Scene Analysis: The Perceptual Organization of Sound. Cambridge, The MIT Press.

28. Sharma, A. and Dorman, M.F. (1999) Cortical auditory evoked potential correlates of categorical perception of voice-onset time. J Acoust Soc Am 106 (2), 1078-83.

29. Traer, J. and McDermott, J.H. (2016) Statistics of natural reverberation enable perceptual separation of sound and space. Proc Natl Acad Sci U S A 113 (48), E7856-E7865.

30. Pandarinath, C. et al. (2010) Symmetry breakdown in the ON and OFF pathways of the 
retina at night: functional implications. J Neurosci 30 (30), 10006-14.

31. Leonhardt, A. et al. (2016) Asymmetry of Drosophila ON and OFF motion detectors enhances real-world velocity estimation. Nat Neurosci 19 (5), 706-715.

32. Green, D.M. (1971) Temporal auditory acuity. Psychol Rev 78 (6), 540-51.

33. Phillips, D.P. et al. (1997) Detection of silent intervals between noises activating different perceptual channels: some properties of "central" auditory gap detection. J Acoust Soc Am 101 (6), 3694-705.

34. Elangovan, S. and Stuart, A. (2008) Natural boundaries in gap detection are related to categorical perception of stop consonants. Ear Hear 29 (5), 761-74.

35. Miller, G.A. and Licklider, J.C.R. (1950) The Intelligibility of Interrupted Speech. The Journal of the Acoustical Society of America 22 (167).

36. Mossbridge, J.A. et al. (2008) Learning and generalization on asynchrony and order tasks at sound offset: implications for underlying neural circuitry. Learn Mem 15 (1), 13-20.

37. Geissler, D.B. and Ehret, G. (2002) Time-critical integration of formants for perception of communication calls in mice. Proc Natl Acad Sci U S A 99 (13), 9021-5.

38. Akimov, A.G. et al. (2017) Spectral summation and facilitation in on- and off-responses for optimized representation of communication calls in mouse inferior colliculus. Eur J Neurosci 45 (3), 440-459.

39. Weible, A.P. et al. (2014) Perceptual gap detection is mediated by gap termination responses in auditory cortex. Curr Biol 24 (13), 1447-55.

40. Pratt, H. et al. (2005) The composite N1 component to gaps in noise. Clin Neurophysiol 116 (11), 2648-63.

41. Casseday, J.H. et al. (1994) Neural tuning for sound duration: role of inhibitory mechanisms in the inferior colliculus. Science 264 (5160), 847-50.

42. Kasai, M. et al. (2012) Distinct neural firing mechanisms to tonal stimuli offset in the inferior colliculus of mice in vivo. Neurosci Res 73 (3), 224-37.

43. Yu, Y.Q. et al. (2004) In vivo intracellular responses of the medial geniculate neurones to acoustic stimuli in anaesthetized guinea pigs. J Physiol 560 (Pt 1), 191-205.

44. He, J. (2001) On and off pathways segregated at the auditory thalamus of the guinea pig. J Neurosci 21 (21), 8672-9.

45. Suga, N. (1964) Single Unit Activity in Cochlear Nucleus and Inferior Colliculus of Echo-Locating Bats. J Physiol 172, 449-74. 
46. Ding, J. et al. (1999) Acoustic and current-pulse responses of identified neurons in the dorsal cochlear nucleus of unanesthetized, decerebrate gerbils. J Neurophysiol 82 (6), 3434-57.

47. Spirou, G.A. and Young, E.D. (1991) Organization of dorsal cochlear nucleus type IV unit response maps and their relationship to activation by bandlimited noise. J Neurophysiol 66 (5), 1750-68.

48. Adams, J.C. and Warr, W.B. (1976) Origins of axons in the cat's acoustic striae determined by injection of horseradish peroxidase into severed tracts. J Comp Neurol 170 (1), 107-21.

49. Shofner, W.P. and Young, E.D. (1985) Excitatory/inhibitory response types in the cochlear nucleus: relationships to discharge patterns and responses to electrical stimulation of the auditory nerve. J Neurophysiol 54 (4), 917-39.

50. Wickesberg, R.E. and Oertel, D. (1990) Delayed, frequency-specific inhibition in the cochlear nuclei of mice: a mechanism for monaural echo suppression. J Neurosci 10 (6), 1762-8.

51. Hancock, K.E. and Voigt, H.F. (1999) Wideband inhibition of dorsal cochlear nucleus type IV units in cat: a computational model. Ann Biomed Eng 27 (1), 73-87.

52. Kulesza, R.J., Jr. et al. (2003) Physiological response properties of neurons in the superior paraolivary nucleus of the rat. J Neurophysiol 89 (4), 2299-312.

53. Yassin, L. et al. (2014) Nitric oxide signaling modulates synaptic inhibition in the superior paraolivary nucleus (SPN) via cGMP-dependent suppression of KCC2. Front Neural Circuits 8,65 .

54. Grothe, B. et al. (2001) Medial superior olive of the big brown bat: neuronal responses to pure tones, amplitude modulations, and pulse trains. J Neurophysiol 86 (5), 2219-30.

55. Felix, R.A., 2nd et al. (2012) Effects of ketamine on response properties of neurons in the superior paraolivary nucleus of the mouse. Neuroscience 201, 307-19.

56. Kopp-Scheinpflug, C. et al. (2011) The sound of silence: ionic mechanisms encoding sound termination. Neuron 71 (5), 911-25.

57. Kulesza, R.J., Jr. et al. (2007) Distinct roles for glycine and GABA in shaping the response properties of neurons in the superior paraolivary nucleus of the rat. J Neurophysiol 97 (2), 1610-20.

58. McCormick, D.A. and Pape, H.C. (1990) Properties of a hyperpolarization-activated cation current and its role in rhythmic oscillation in thalamic relay neurones. J Physiol 431, 
291-318.

59. Anderson, T.M. and Ramirez, J.M. (2017) Respiratory rhythm generation: triple oscillator hypothesis. F1000Res 6, 139.

60. Dodla, R. et al. (2006) Well-timed, brief inhibition can promote spiking: postinhibitory facilitation. J Neurophysiol 95 (4), 2664-77.

61. Beiderbeck, B. et al. (2018) Precisely timed inhibition facilitates action potential firing for spatial coding in the auditory brainstem. Nat Commun 9 (1), 1771.

62. Lohrke, S. et al. (2005) Shift from depolarizing to hyperpolarizing glycine action occurs at different perinatal ages in superior olivary complex nuclei. Eur J Neurosci 22 (11), 2708-22.

63. Felix, R.A., 2nd et al. (2011) Sound rhythms are encoded by postinhibitory rebound spiking in the superior paraolivary nucleus. J Neurosci 31 (35), 12566-78.

64. Roberts, R.C. and Ribak, C.E. (1987) GABAergic neurons and axon terminals in the brainstem auditory nuclei of the gerbil. J Comp Neurol 258 (2), 267-80.

65. Kulesza, R.J., Jr. and Berrebi, A.S. (2000) Superior paraolivary nucleus of the rat is a GABAergic nucleus. J Assoc Res Otolaryngol 1 (4), 255-69.

66. Schofield, B.R. et al. (2014) Subcollicular projections to the auditory thalamus and collateral projections to the inferior colliculus. Front Neuroanat 8, 70.

67. Xie, R. et al. (2007) Rethinking tuning: in vivo whole-cell recordings of the inferior colliculus in awake bats. J Neurosci 27 (35), 9469-81.

68. Vater, M. et al. (1992) The functional role of GABA and glycine in monaural and binaural processing in the inferior colliculus of horseshoe bats. J Comp Physiol A 171 (4), 541-53.

69. Sanchez, J.T. et al. (2008) Glycinergic "inhibition" mediates selective excitatory responses to combinations of sounds. J Neurosci 28 (1), 80-90.

70. 55

, R., II et al. (2014) The superior paraolivary nucleus shapes temporal response properties of neurons in the inferior colliculus. Brain Structure and Function, 1-14.

71. He, J. (2003) Corticofugal modulation on both ON and OFF responses in the nonlemniscal auditory thalamus of the guinea pig. J Neurophysiol 89 (1), 367-81.

72. Yu, Y.Q. et al. (2004) Corticofugal gating of auditory information in the thalamus: an in vivo intracellular recording study. J Neurosci 24 (12), 3060-9.

73. Bartlett, E.L. and Smith, P.H. (1999) Anatomic, intrinsic, and synaptic properties of dorsal and ventral division neurons in rat medial geniculate body. J Neurophysiol 81 (5), 
1999-2016.

74. Wang, X.X. et al. (2016) Characterization of Rebound Depolarization in Neurons of the Rat Medial Geniculate Body In Vitro. Neurosci Bull 32 (1), 16-26.

75. Lee, C.C. and Sherman, S.M. (2010) Topography and physiology of ascending streams in the auditory tectothalamic pathway. Proc Natl Acad Sci U S A 107 (1), 372-7.

76. Hu, B. (1995) Cellular basis of temporal synaptic signalling: an in vitro electrophysiological study in rat auditory thalamus. J Physiol 483 ( Pt 1), 167-82.

77. Polley, D.B. et al. (2007) Multiparametric auditory receptive field organization across five cortical fields in the albino rat. J Neurophysiol 97 (5), 3621-38.

78. Coomes Peterson, D. and Schofield, B.R. (2007) Projections from auditory cortex contact ascending pathways that originate in the superior olive and inferior colliculus. Hear Res $232(1-2), 67-77$.

79. Recanzone, G.H. (2000) Response profiles of auditory cortical neurons to tones and noise in behaving macaque monkeys. Hear Res 150 (1-2), 104-18.

80. Takahashi, H. et al. (2004) Cortical mapping of auditory-evoked offset responses in rats. Neuroreport 15 (10), 1565-9.

81. Qin, L. et al. (2007) Comparison between offset and onset responses of primary auditory cortex ON-OFF neurons in awake cats. J Neurophysiol 97 (5), 3421-31.

82. Keller, C.H. et al. (2018) Gap encoding by parvalbumin-expressing interneurons in auditory cortex. Journal of Neurophysiology.

83. Tian, B. et al. (2013) Analogues of simple and complex cells in rhesus monkey auditory cortex. Proc Natl Acad Sci U S A 110 (19), 7892-7.

84. Volkov, I.O. and Galazjuk, A.V. (1991) Formation of spike response to sound tones in cat auditory cortex neurons: interaction of excitatory and inhibitory effects. Neuroscience 43 $(2-3), 307-21$.

85. Ramamurthy, D.L. and Recanzone, G.H. (2017) Spectral and spatial tuning of onset and offset response functions in auditory cortical fields $A 1$ and $C L$ of rhesus macaques. J Neurophysiol 117 (3), 966-986.

86. Kusmierek, P. and Rauschecker, J.P. (2009) Functional specialization of medial auditory belt cortex in the alert rhesus monkey. J Neurophysiol 102 (3), 1606-22.

87. Chimoto, S. et al. (2002) Tonal response patterns of primary auditory cortex neurons in alert cats. Brain Res 934 (1), 34-42. 
88. Hartley, D.E. et al. (2011) Binaural sensitivity changes between cortical on and off responses. J Neurophysiol 106 (1), 30-43.

89. Moshitch, D. et al. (2006) Responses of neurons in primary auditory cortex (A1) to pure tones in the halothane-anesthetized cat. J Neurophysiol 95 (6), 3756-69.

90. Campbell, R.A. et al. (2010) Brief sounds evoke prolonged responses in anesthetized ferret auditory cortex. J Neurophysiol 103 (5), 2783-93.

91. Baba, H. et al. (2016) Auditory cortical field coding long-lasting tonal offsets in mice. Sci Rep 6, 34421.

92. Fishman, Y.I. and Steinschneider, M. (2009) Temporally dynamic frequency tuning of population responses in monkey primary auditory cortex. Hear Res 254 (1-2), 64-76.

93. Kato, H.K. et al. (2015) Flexible Sensory Representations in Auditory Cortex Driven by Behavioral Relevance. Neuron 88 (5), 1027-1039.

94. Xiong, X.R. et al. (2013) Interaural level difference-dependent gain control and synaptic scaling underlying binaural computation. Neuron 79 (4), 738-53.

95. Suga, N. et al. (1975) Peripheral specialization for fine analysis of doppler-shifted echoes in the auditory system of the "CF-FM" bat Pteronotus parnellii. J Exp Biol 63 (1), 161-92.

96. Geisler, C.D. and Sinex, D.G. (1982) Responses of primary auditory fibers to brief tone bursts. J Acoust Soc Am 72 (3), 781-94.

97. Henry, K.R. (1988) Effects of acoustic and sensory variables on masking tuning curves of the offset auditory brain-stem response in the rodent. Electroencephalogr Clin Neurophysiol 69 (5), 476-85.

98. Rhode, W.S. and Smith, P.H. (1985) Characteristics of tone-pip response patterns in relationship to spontaneous rate in cat auditory nerve fibers. Hear Res 18 (2), 159-68.

99. Lewis, E.R. and Henry, K.R. (1989) Transient responses to tone bursts. Hear Res 37 (3), 219-39.

100. Zheng, J. et al. (2011) Persistence of past stimulations: storing sounds within the inner ear. Biophys J 100 (7), 1627-34.

101. Pedersen, C.B. and Salomon, G. (1977) Temporal integration of acoustic energy. Acta Otolaryngol 83 (5-6), 417-23.

102. Fuzessery, Z.M. and Hall, J.C. (1999) Sound duration selectivity in the pallid bat inferior colliculus. Hear Res 137 (1-2), 137-54.

103. Leary, C.J. et al. (2008) Midbrain auditory neurons integrate excitation and inhibition to 
generate duration selectivity: an in vivo whole-cell patch study in anurans. J Neurosci 28 (21), 5481-93.

104. Narins, P.M. and Capranica, R.R. (1980) Neural adaptations for processing the two-note call of the Puerto Rican treefrog, Eleutherodactylus coqui. Brain Behav Evol 17 (1), 48-66.

105. He, J. et al. (1997) Temporal integration and duration tuning in the dorsal zone of cat auditory cortex. J Neurosci 17 (7), 2615-25.

106. Perez-Gonzalez, D. et al. (2006) Duration selective neurons in the inferior colliculus of the rat: topographic distribution and relation of duration sensitivity to other response properties. J Neurophysiol 95 (2), 823-36.

107. Brand, A. et al. (2000) Duration tuning in the mouse auditory midbrain. J Neurophysiol 84 (4), 1790-9.

108. Belin, P. et al. (2002) The neuroanatomical substrate of sound duration discrimination. Neuropsychologia 40 (12), 1956-64.

109. Kadner, A. et al. (2006) Neurons in the medial nucleus of the trapezoid body and superior paraolivary nucleus of the rat may play a role in sound duration coding. J Neurophysiol 95 (3), 1499-508.

110. Qin, L. et al. (2009) Neural and behavioral discrimination of sound duration by cats. J Neurosci 29 (50), 15650-9.

111. Phillips, S.L. et al. (1994) Auditory duration discrimination in young and elderly listeners with normal hearing. J Am Acad Audiol 5 (3), 210-5.

112. Portfors, C.V. (2007) Types and functions of ultrasonic vocalizations in laboratory rats and mice. J Am Assoc Lab Anim Sci 46 (1), 28-34.

113. Soskuthy, M. and Hay, J. (2017) Changing word usage predicts changing word durations in New Zealand English. Cognition 166, 298-313.

114. Rammsayer, T.H. and Lima, S.D. (1991) Duration discrimination of filled and empty auditory intervals: cognitive and perceptual factors. Percept Psychophys 50 (6), 565-74.

115. Pratt, H. et al. (2008) The auditory P50 component to onset and offset of sound. Clin Neurophysiol 119 (2), 376-87.

116. Hooper, S.L. et al. (2002) A computational role for slow conductances: single-neuron models that measure duration. Nat Neurosci 5 (6), 552-6.

117. Robin, D.A. and Royer, F.L. (1987) Auditory temporal processing: two-tone flutter fusion and a model of temporal integration. J Acoust Soc Am 82 (4), 1207-17. 
118. Abel, S.M. (1972) Duration discrimination of noise and tone bursts. J Acoust Soc Am 51 (4), 1219-23.

119. Sayegh, R. et al. (2011) Duration tuning in the auditory midbrain of echolocating and non-echolocating vertebrates. J Comp Physiol A Neuroethol Sens Neural Behav Physiol 197 (5), 571-83.

120. Alluri, R.K. et al. (2016) Phasic, suprathreshold excitation and sustained inhibition underlie neuronal selectivity for short-duration sounds. Proc Natl Acad Sci U S A 113 (13), E1927-35.

121. Aubie, B. et al. (2009) Computational models of millisecond level duration tuning in neural circuits. J Neurosci 29 (29), 9255-70.

122. Schneider, B.A. et al. (1994) Gap detection and the precedence effect in young and old adults. J Acoust Soc Am 95 (2), 980-91.

123. Snell, K.B. and Frisina, D.R. (2000) Relationships among age-related differences in gap detection and word recognition. J Acoust Soc Am 107 (3), 1615-26.

124. Gordon-Salant, S. and Fitzgibbons, P.J. (1993) Temporal factors and speech recognition performance in young and elderly listeners. J Speech Hear Res 36 (6), 1276-85.

125. Pichora-Fuller, M.K. et al. (2007) Temporal jitter disrupts speech intelligibility: a simulation of auditory aging. Hear Res 223 (1-2), 114-21.

126. Harris, K.C. et al. (2012) Human evoked cortical activity to silent gaps in noise: effects of age, attention, and cortical processing speed. Ear Hear 33 (3), 330-9.

127. Skoe, E. et al. (2015) Stability and plasticity of auditory brainstem function across the lifespan. Cereb Cortex 25 (6), 1415-26.

128. Phillips, D.P. et al. (2010) Auditory temporal gap detection in children with and without auditory processing disorder. J Am Acad Audiol 21 (6), 404-8.

129. Johnson, K.L. et al. (2005) Brain stem response to speech: a biological marker of auditory processing. Ear Hear 26 (5), 424-34.

130. Caspary, D.M. et al. (2008) Inhibitory neurotransmission, plasticity and aging in the mammalian central auditory system. J Exp Biol 211 (Pt 11), 1781-91.

131. Chambers, A.R. et al. (2016) Central Gain Restores Auditory Processing following Near-Complete Cochlear Denervation. Neuron 89 (4), 867-79.

132. Sturm, J.J. et al. (2017) Noise Trauma-Induced Behavioral Gap Detection Deficits Correlate with Reorganization of Excitatory and Inhibitory Local Circuits in the Inferior Colliculus and 
Are Prevented by Acoustic Enrichment. J Neurosci 37 (26), 6314-6330.

133. Libe-Philippot, B. et al. (2017) Auditory cortex interneuron development requires cadherins operating hair-cell mechanoelectrical transduction. Proc Natl Acad Sci U S A 114 (30), 7765-7774. 


\section{Highlights}

2

3 - Sound offsets are important cues for perceptual grouping, duration discrimination, gap detection and consonant identification.

- Sound-offset responses are found throughout the auditory brain and are also concentrated in particular subregions, suggesting the existence of an "offset pathway" in the central auditory system.

- In the auditory brainstem, sound-offset responses are generated de novo and independently in both the dorsal cochlear nucleus and the superior paraolivary nucleus.

- In the auditory midbrain and thalamus, sound-offset responses might be either inherited from the brainstem, generated de novo, or both. In the cortex, soundoffset responses appear to be inherited from ascending pathways.

14 - Specific deficits in sound-offset responses have been reported in animal models, and might contribute to auditory temporal processing deficits in aging and disease. 


\section{Outstanding Questions}

- In natural sounds, offsets are typically slower than onsets and obscured by reverberation, although detailed statistics of onset/offset asymmetries in natural environments remain to be determined. In the central auditory system, soundoffset responses are less prevalent than sound-onset responses, and the two types of responses often differ in frequency tuning or spatial tuning. Do onset/offset asymmetries in the auditory system reflect efficient coding of natural sound statistics? And what are the mechanistic origins and functional significance of onset/offset asymmetries in frequency tuning and spatial tuning?

- Gap-detection tests are widely used (in the clinic as well as in the lab) as a measure of the limits of auditory temporal acuity. However, the relative contributions of sound-offset and sound-onset responses to gap detection are unclear, and the neural circuits underlying within-channel and between-channel gap detection are still poorly understood. What is the role of sound-offset responses in gap detection?

- The mechanisms of offset-response generation in the auditory brainstem are well understood for SPN neurons, but not for DCN neurons. Offset responses have been observed in DCN Type III and IV neurons, which receive more extensive synaptic inhibition than other DCN cell types. Evidence suggests that offset responses in the DCN might be generated by post-inhibitory rebound, but the details of the cellular mechanism remain unknown. How are sound-offset responses generated in the cochlear nucleus?

Sound-offset responses in the IC, MGB, and AC all appear to be at least partly inherited from brainstem generators in the DCN and/or SPN. However, there is also some evidence for de novo generation or amplification of offset responses in these areas. What are the cellular and/or circuit mechanisms driving offset responses in the auditory midbrain, thalamus, and cortex? 
- Sound-offset responses may be particularly sensitive to disruption of excitationinhibition balance in aging and disease. Specific abnormalities in sound-offset responses have been reported in animal models, but the perceptual consequences of these abnormalities are still unknown. Do impairments in sound-offset sensitivity contribute to auditory dysfunction in human aging and disease? 


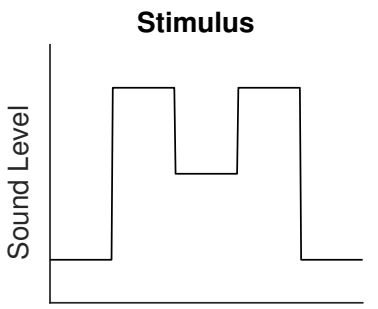

Time

B

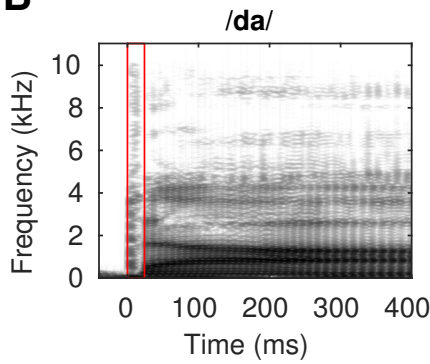

C

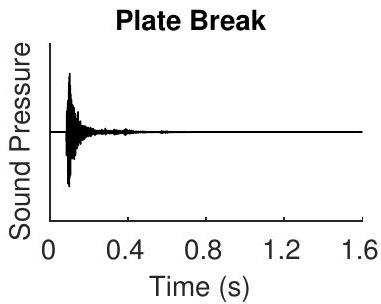

Single Pathway

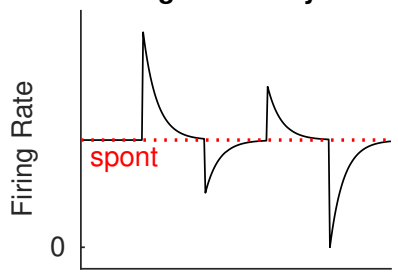

Time
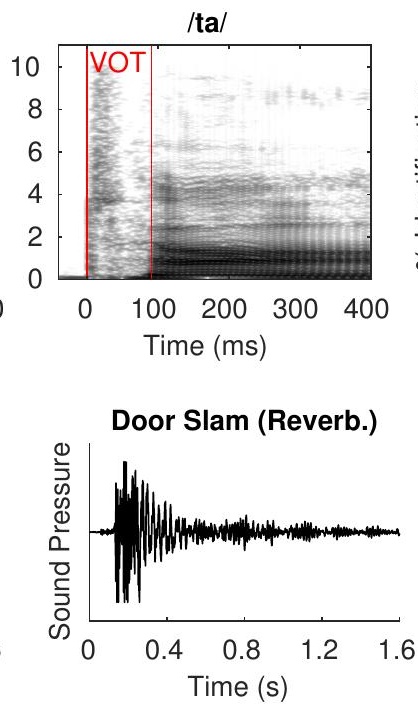

Dual Pathways

0

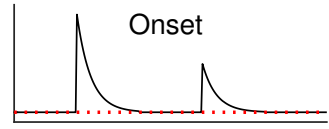

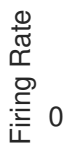
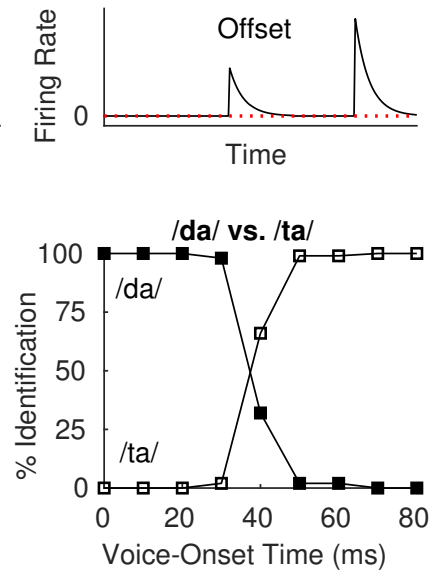

Speech

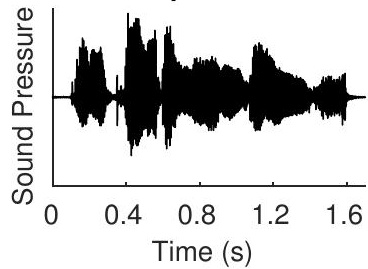




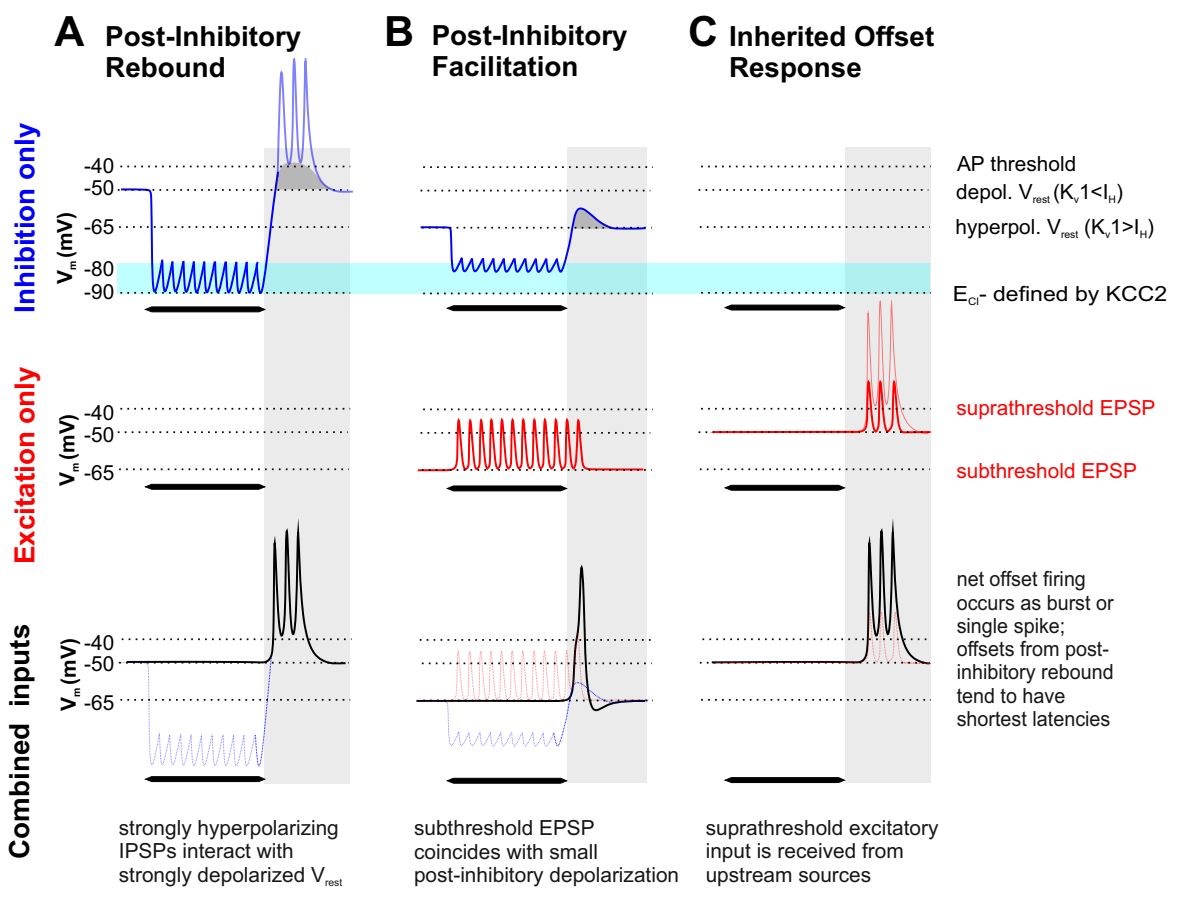


A Sound-Offset Pathway
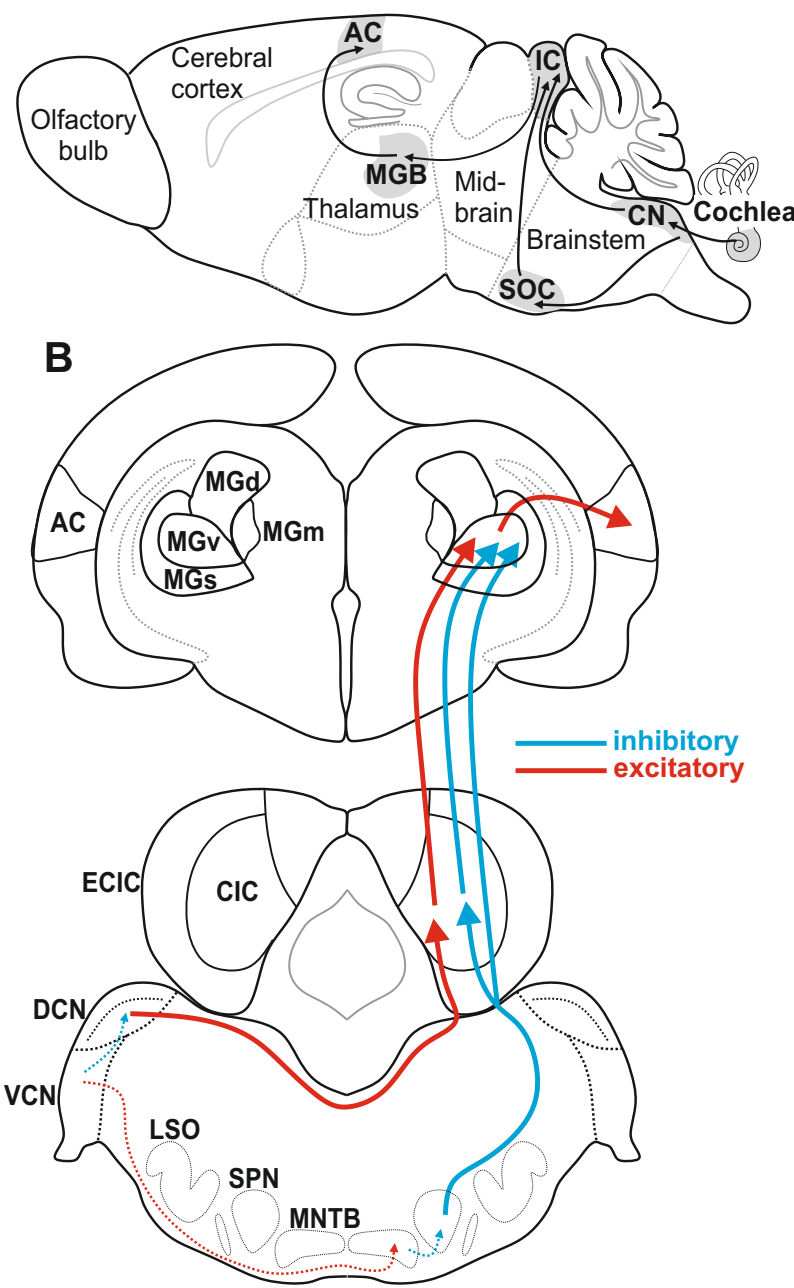

Superior olivary complex
C
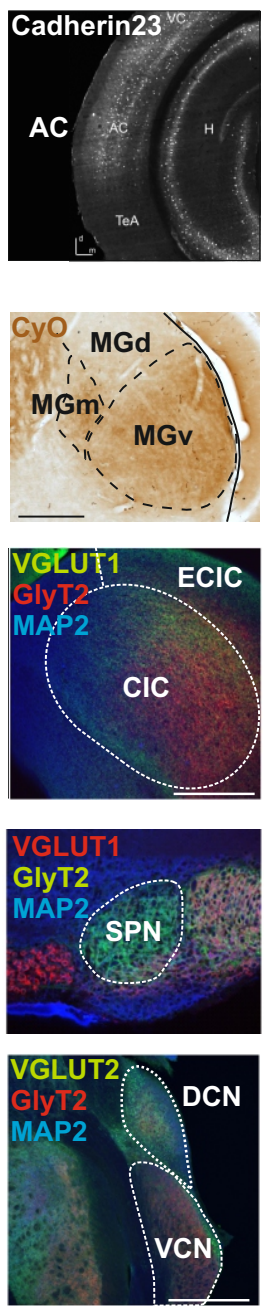

D Sound-Offset Responses
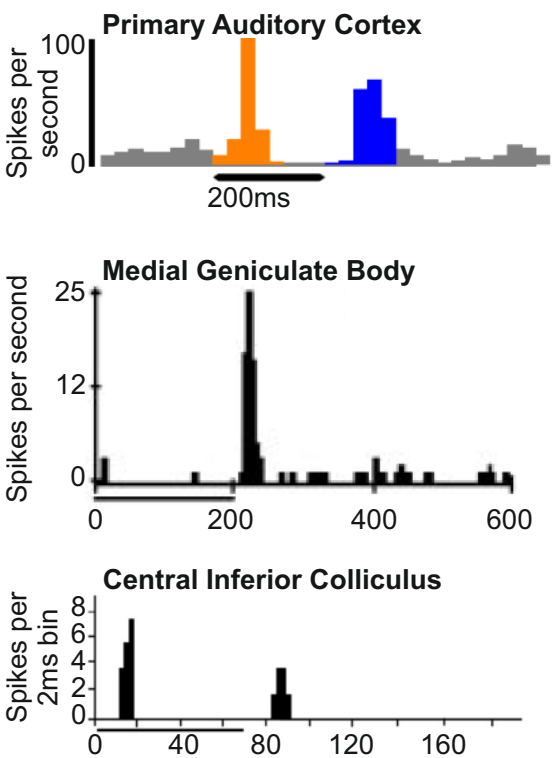

둥 444 Superior Paraolivary Nucleus
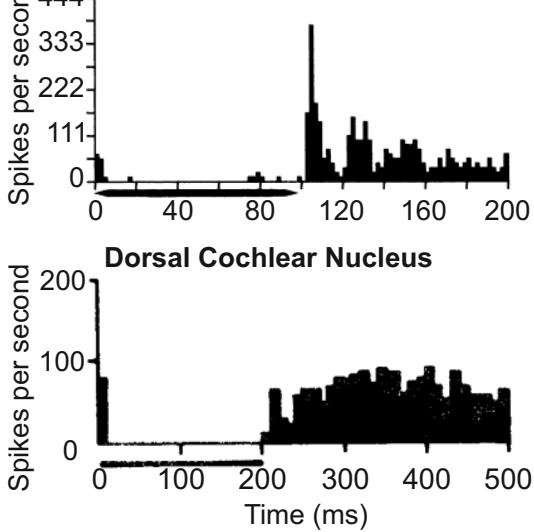